\title{
Krylov Subspace Spectral Methods for the Time-Dependent Schrödinger Equation with Non-Smooth Potentials
}

\author{
James V. Lambers *
}

\begin{abstract}
This paper presents modifications of Krylov Subspace Spectral (KSS) Methods, which build on the work of Gene Golub and others pertaining to moments and Gaussian quadrature to produce high-order accurate approximate solutions to the time-dependent Schrödinger equation in the case where either the potential energy or the initial data is not a smooth function. These modifications consist of using various symmetric perturbations to compute off-digonal elements of functions of matrices. It is demonstrated through analytical and numerical results that KSS methods, with these modifications, achieve the same high-order accuracy and possess the same stability properties as they do when applied to parabolic problems, even though the solutions to the Schrödinger equation do not possess the same smoothness.
\end{abstract}

Keywords: spectral methods, Gaussian quadrature, Schrödinger equation, variable-coefficient, Lanczos method

\section{Introduction}

Consider the following initial-boundary value problem in one space dimension,

$$
\begin{gathered}
u_{t}+i L u=0 \quad \text { on }(0,2 \pi) \times(0, \infty), \\
u(x, 0)=f(x), \quad 0<x<2 \pi,
\end{gathered}
$$

with periodic boundary conditions

$$
u(0, t)=u(2 \pi, t), \quad t>0 .
$$

The operator $L$ is a second-order differential operator of the form

$$
L u=-p u_{x x}+V(x) u,
$$

where $p$ is a positive constant and $V(x)$ is a nonnegative (but nonzero) smooth function. It follows that $L$ is selfadjoint and positive definite.

\footnotetext{
* Submitted January 12, 2010. matics, University of Southern Mississippi, Hattiesburg, MS 39406-0001, USA Tel/Fax: 691-266-5784/5818 Email: James.Lambers@usm.edu
}

This equation is a simplification of the time-dependent Schrödinger equation,

$$
i \hbar \frac{\partial \psi}{\partial t}=-\frac{\hbar^{2}}{2 m} \nabla^{2} \psi+V \psi
$$

that predicts the future behavior of a dynamic system. The constant $\hbar$ is Planck's constant, $m$ is mass, $V$ is potential energy, and the solution $\psi$ is a wavefunction that describes the quantum state of an electron [16]. By restricting ourselves to one space dimension, and using the transformation $\tau=t / \hbar$, we obtain (1) with $p=\hbar^{2} / 2 m$.

In [14] a class of methods, called Krylov subspace spectral (KSS) methods, was introduced for the purpose of solving parabolic variable-coefficient problems such as those of the form $u_{t}+L u=0$. These methods are based on the application of techniques developed by Golub and Meurant in [7], originally for the purpose of computing elements of the inverse of a matrix, to elements of the matrix exponential of an operator. It has been shown in these references that KSS methods, by employing different approximations of the solution operator for each Fourier component of the solution, achieve higher-order accuracy in time than other Krylov subspace methods (see, for example, [10]) for stiff systems of ODE, and, as shown in [11], they are also quite stable, considering that they are explicit methods.

In this paper, we explore the application of KSS methods to the time-dependent Schrödinger equation. Unlike the heat equation, whose solutions are infinitely differentiable, solutions of (1), (2), (3) retain the smoothness of the initial data. This property makes these problems more difficulty for spectral methods, as they must accurately resolve a larger number of Fourier components. This difficulty is substantially increased if the potential $V(x)$ exhibits rough behavior such as oscillations or discontinuities. We will find that KSS methods, as described in the abovementioned references, are not very effective for such problems, but can easily be modified in order to achieve the same high-order accuracy and stability as for problems with a smooth potential.

Section 2 reviews the main properties of KSS methods, including algorithmic details and results concerning lo- 
cal accuracy. In Section 3, we discuss the application of KSS methods to the Schrödinger equation and present some results about its convergence behavior. We then show how they can be modified to most effectively handle problems with a potential or initial data that is not smooth, and indicate why such a modification is effective. Numerical results are presented in Section 4. In Section 5 , future directions are discussed.

\section{Krylov Subspace Spectral Methods}

We begin with a review of the main aspects of KSS methods. Let $S(t)=\exp [-L t]$ represent the exact solution operator of the problem $u_{t}+L u=0$, with initial condition $(2)$, and boundary condition $(3)$, and let $\langle\cdot, \cdot\rangle$ denote the standard inner product of functions defined on $[0,2 \pi]$.

Krylov subspace spectral methods, introduced in [14], use Gaussian quadrature on the spectral domain to compute the Fourier components of the solution. These methods are time-stepping algorithms that compute the solution at time $t_{1}, t_{2}, \ldots$, where $t_{n}=n \Delta t$ for some choice of $\Delta t$. Given the computed solution $\tilde{u}\left(x, t_{n}\right)$ at time $t_{n}$, the solution at time $t_{n+1}$ is computed by approximating the Fourier components that would be obtained by applying the exact solution operator to $\tilde{u}\left(x, t_{n}\right)$,

$$
\hat{u}\left(\omega, t_{n+1}\right)=\left\langle\frac{1}{\sqrt{2 \pi}} e^{i \omega x}, S(\Delta t) \tilde{u}\left(x, t_{n}\right)\right\rangle .
$$

We discretize functions defined on $[0,2 \pi]$ on an $N$-point uniform grid with spacing $\Delta x=2 \pi / N$. With this discretization, the operator $L$ and the solution operator $S(\Delta t)$ can be approximated by $N \times N$ matrices that represent linear operators on the space of grid functions, and the quantity (6) can be approximated by a bilinear form

$$
\hat{u}\left(\omega, t_{n+1}\right) \approx \hat{\mathbf{e}}_{\omega}^{H} S_{N}(\Delta t) \mathbf{u}\left(t_{n}\right),
$$

where

$$
\left[\hat{\mathbf{e}}_{\omega}\right]_{j}=\frac{1}{\sqrt{2 \pi}} e^{i \omega j \Delta x}, \quad\left[\mathbf{u}\left(t_{n}\right)\right]_{j}=u\left(j \Delta x, t_{n}\right),
$$

and

$$
S_{N}(t)=\exp \left[-L_{N} t\right], \quad\left[L_{N}\right]_{j k}=-p\left[D_{N}^{2}\right]_{j k}+q(j \Delta x)
$$

where $D_{N}$ is a discretization of the differentiation operator that is defined on the space of grid functions. Our goal is to approximate (7) by computing an approximation to

$$
\left[\hat{\mathbf{u}}^{n+1}\right]_{\omega}=\hat{\mathbf{e}}_{\omega}^{H} \mathbf{u}\left(t_{n+1}\right)=\hat{\mathbf{e}}_{\omega}^{H} S_{N}(\Delta t) \mathbf{u}\left(t_{n}\right)
$$

In [7] Golub and Meurant describe a method for computing quantities of the form

$$
\mathbf{u}^{T} f(A) \mathbf{v}
$$

where $\mathbf{u}$ and $\mathbf{v}$ are $N$-vectors, $A$ is an $N \times N$ symmetric positive definite matrix, and $f$ is a smooth function. Our goal is to apply this method with $A=L_{N}$ where $L_{N}$ was defined in $(9), f(\lambda)=\exp (-\lambda t)$ for some $t$, and the vectors $\mathbf{u}$ and $\mathbf{v}$ are derived from $\hat{\mathbf{e}}_{\omega}$ and $\mathbf{u}\left(t_{n}\right)$.

The basic idea is as follows: since the matrix $A$ is symmetric positive definite, it has real eigenvalues

$$
b=\lambda_{1} \geq \lambda_{2} \geq \cdots \geq \lambda_{N}=a>0,
$$

and corresponding orthogonal eigenvectors $\mathbf{q}_{j}, j=$ $1, \ldots, N$. Therefore, the quantity (11) can be rewritten as

$$
\mathbf{u}^{T} f(A) \mathbf{v}=\sum_{\ell=1}^{N} f\left(\lambda_{\ell}\right) \mathbf{u}^{T} \mathbf{q}_{j} \mathbf{q}_{j}^{T} \mathbf{v} .
$$

We let $a=\lambda_{N}$ be the smallest eigenvalue, $b=\lambda_{1}$ be the largest eigenvalue, and define the measure $\alpha(\lambda)$ by

$$
\alpha(\lambda)= \begin{cases}0, & \text { if } \lambda<a \\ \sum_{j=i}^{N} \alpha_{j} \beta_{j}, & \text { if } \lambda_{i} \leq \lambda<\lambda_{i-1} \\ \sum_{j=1}^{N} \alpha_{j} \beta_{j}, & \text { if } b \leq \lambda\end{cases}
$$

where $\alpha_{j}=\mathbf{u}^{T} \mathbf{q}_{j}$ and $\beta_{j}=\mathbf{q}_{j}^{T} \mathbf{v}$. If this measure is positive and increasing, then the quantity (11) can be viewed as a Riemann-Stieltjes integral

$$
\mathbf{u}^{T} f(A) \mathbf{v}=I[f]=\int_{a}^{b} f(\lambda) d \alpha(\lambda) .
$$

As discussed in [4], [5], [6], [7], the integral $I[f]$ can be bounded using either Gauss, Gauss-Radau, or GaussLobatto quadrature rules, all of which yield an approximation of the form

$$
I[f]=\sum_{j=1}^{K} w_{j} f\left(t_{j}\right)+R[f],
$$

where the nodes $t_{j}, j=1, \ldots, K$, as well as the weights $w_{j}, j=1, \ldots, K$, can be obtained using the symmetric Lanczos algorithm if $\mathbf{u}=\mathbf{v}$, and the unsymmetric Lanczos algorithm if $\mathbf{u} \neq \mathbf{v}$ ( see $[9]$ ).

In the case $\mathbf{u} \neq \mathbf{v}$, there is the possibility that the weights may not be positive, which destabilizes the quadrature rule (see [1] for details). Therefore, it is best to handle this case by rewriting (11) using decompositions such as

$$
\mathbf{u}^{T} f(A) \mathbf{v}=\frac{1}{\delta}\left[\mathbf{u}^{T} f(A)(\mathbf{u}+\delta \mathbf{v})-\mathbf{u}^{T} f(A) \mathbf{u}\right],
$$

where $\delta$ is a small constant. Guidelines for choosing an appropriate value for $\delta$ can be found in [14, Section 2.2].

Employing these quadrature rules yields the following basic process (for details see [14]) for computing the Fourier coefficients of $\mathbf{u}\left(t_{n+1}\right)$ from $\mathbf{u}\left(t_{n}\right)$. It is assumed that 
when the Lanczos algorithm (symmetric or unsymmetric) is employed, $K$ iterations are performed to obtain the $K$ quadrature nodes and weights.

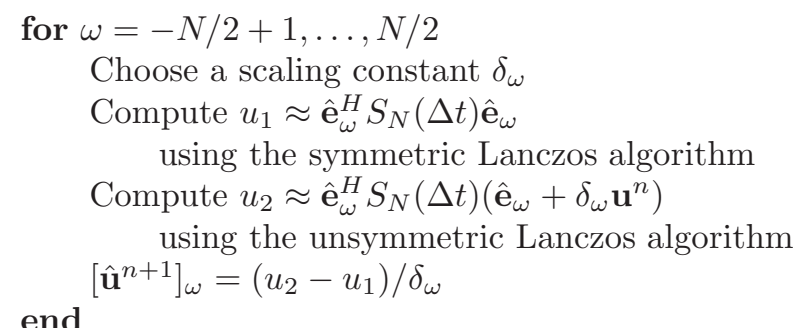

It should be noted that the constant $\delta_{\omega}$ plays the role of $\delta$ in the decomposition (17), and the subscript $\omega$ is used to indicate that a different value may be used for each wave number $\omega=-N / 2+1, \ldots, N / 2$. Also, in the presentation of this algorithm in [14], a polar decomposition is used instead of (17), and is applied to sines and cosines instead of complex exponential functions.

If we let $\delta_{\omega} \rightarrow 0$ for each $\omega$, it follows from [7] that the leading term in the quadrature error for each Fourier component is

$\left.\frac{\Delta t^{2 K}}{(2 K) !} \frac{d}{d \delta_{\omega}}\left[\hat{\mathbf{e}}_{\omega}^{H} \prod_{j=1}^{K}\left(L_{N}-\lambda_{j, \omega}\left(\delta_{\omega}\right) I\right)^{2}\left(\hat{\mathbf{e}}_{\omega}+\delta_{\omega} \mathbf{u}^{n}\right)\right]\right|_{\delta_{\omega}=0}$.

where, for $j=1, \ldots, K, \lambda_{j, \omega}\left(\delta_{\omega}\right)$ is the $j$ th Gaussian quadrature node obtained by applying the Lanczos algorithm to $L_{N}$ with starting vectors $\hat{\mathbf{e}}_{\omega}$ and $\hat{\mathbf{e}}_{\omega}+\delta_{\omega} \mathbf{u}^{n}$.

Carrying out the differentiation, we obtain

$$
\frac{\Delta t^{2 K}}{(2 K) !} \hat{\mathbf{e}}_{\omega}^{H} \prod_{j=1}^{K}\left(L_{N}-t_{j, \omega}(0) I\right)^{2} \mathbf{u}^{n}
$$

as the choice of Gaussian nodes causes all other terms arising from the use of the product rule to vanish. This error term suggests that KSS methods achieve high-order temporal accuracy, as the constant multiplying $\Delta t^{2 K}$ is a Fourier coefficient of the application of a pseudodifferential operator of order $4 K$ to $\mathbf{u}^{n}$. Given sufficient regularity of the solution, it can be shown that KSS methods are consistent, and actually do achieve this high-order accuracy, as already demonstrated in numerical results in $[11,12,14,15]$.

On the surface, it may appear to be a simple matter to generalize KSS methods to a problem such as the timedependent Schrödinger equation, since the solution process differs from that used for parabolic equations only by a change of solution operator, which is reflected by a change of integrand in (15). While this trivial adjustment is sufficient for problems in which the variable coefficient, the potential $V(x)$, and the initial data $f(x)$ are smooth, the resulting method is much less effective when this is not the case. We must therefore investigate if some modification of KSS methods can address these difficulties.

\section{Application to the Schrödinger Equa- tion}

It is straightforward to modify KSS methods applied to problems of the form $u_{t}+L u=0$ to a problem of the form (1): it is only necessary to change the integrand for each Riemann-Stieltjes integral from $e^{-\lambda t}$ to $e^{-i \lambda t}$.

\subsection{Convergence Analysis}

When $K=1$, the approximate solution operator has a particularly simple form, which yields the following results. We will denote by $\tilde{S}_{N}(\Delta t) f$ the result of applying a single time step of a KSS method to the function $f(x)$, using a discretization of space and time with uniform spacings $\Delta x=2 \pi / N$ and $\Delta t$, respectively. Also, for convenience, component-wise multiplication of vectors $\mathbf{u}$ and $\mathbf{v}$ is written as simply $\mathbf{u v}$.

Theorem 3.1 Let $V(x)$ in (4) belong to $B L_{M}([0,2 \pi])$ for a fixed integer $M$. Let $f \in H_{p}^{q}[0,2 \pi]$, for $q \geq 5$. Then, for the problem (1), (2), (3), on the domain $[0,2 \pi] \times$ $[0, T]$, the one-node KSS method is consistent. That is,

$$
\left\|\tilde{S}_{N}(\Delta t) f_{N}-\exp [-i L \Delta t] f\right\|_{L^{2}} \leq C_{1} \Delta t^{2}+C_{2} \Delta x^{q},
$$

where $f_{N}(x)=P_{N} f(x)$, and the constants $C_{1}$ and $C_{2}$ are independent of $\Delta x$ and $\Delta t$.

Now we consider the stability of the method. For convenience, we denote by $\tilde{S}_{N}(\Delta t)$ the matrix such that $\mathbf{u}^{n+1}=\tilde{S}_{N}(\Delta t) \mathbf{u}^{n}$, for given $N$ and $\Delta t$.

Theorem 3.2 Let $V(x)$ in (4) belong to $B L_{M}([0,2 \pi])$ for a fixed integer $M$. Then, for the problem (1), (2), (3), the one-node KSS method is unconditionally stable. That is, given $T>0$, there exists a constant $C_{T}$, independent of $N$ and $\Delta t$, such that

$$
\left\|\tilde{S}_{N}(\Delta t)^{n}\right\|_{2} \leq C_{T},
$$

for $0 \leq n \Delta t \leq T$.

The Schrödinger equation is unitary, which means that the solution $u(x, t)$ satisfies

$$
\frac{d}{d t}\left[\int_{0}^{2 \pi}|u(x, t)|^{2} d x\right]=0 .
$$

It is natural to ask whether numerical solutions computed by KSS methods also satisfy this property. For the 1-node case, we have the following result. 
Proceedings of the International MultiConference of Engineers and Computer Scientists $2010 \mathrm{Vol}$ III, IMECS 2010, March 17 - 19, 2010, Hong Kong

Theorem 3.3 Let $\mathbf{u}^{n}$ be the approximate solution of (1), (2), (3) by the one-node KSS method on an N-point grid. Then

$$
\left\|\mathbf{u}^{n+1}\right\|^{2}=\left\|\mathbf{u}^{n}\right\|^{2}+O\left(\Delta t^{2}\right) .
$$

That is, the one-node KSS method is unitary in the limit as $\Delta t \rightarrow 0$.

Intuitively, one would expect this result as a natural consequence of the consistency of the scheme, but it should also be noted that the departure from unitarity is directly proportional to the heterogeneity in the potential $V(x)$.

Now we can prove that the method converges. For convenience, we define the 2 -norm of a function $u(x, t)$ to be the vector 2 -norm of the restriction of $u(x, t)$ to the spatial grid:

$$
\|u(\cdot, t)\|_{2}=\left(\sum_{j=0}^{N-1}|u(j \Delta x, t)|^{2}\right)^{1 / 2}
$$

We also say that a method is convergent of order $(m, n)$ if there exist constants $C_{t}$ and $C_{x}$, independent of the time step $\Delta t$ and grid spacing $\Delta x=2 \pi / N$, such that

$$
\left\|u(\cdot, t)-\tilde{u}_{N}(\cdot, t)\right\|_{2} \leq C_{t} \Delta t^{m}+C_{x} \Delta x^{n}, \quad 0 \leq t \leq T .
$$

Theorem 3.4 Let $V(x)$ in (4) belong to $B L_{M}([0,2 \pi])$ for some integer $M$. Let $f \in H_{p}^{q}[0,2 \pi]$, where $q \geq 5$. Then, for the problem (1), (2), (3), on the domain $[0,2 \pi] \times[0, T]$, the one-node KSS method is convergent of order $(1, q)$.

Theorem 3.1 can be generalized to the case of more than one quadrature node per Fourier coefficient, in which case the temporal error is $O\left(\Delta t^{2 K}\right)$, where $K$ is the number of quadrature nodes in each rule. This entails expanding each Fourier coefficient of the approximate solution, in a Taylor series around $\Delta t=0$, and then establishing bounds on the nodes $\lambda_{\omega, j}$ and weights $w_{\omega, j}$, and their derivatives with respect to $\delta_{\omega}$, at $\delta_{\omega}=0$, for $j=1, \ldots, K$ and $\omega=-N / 2+1, \ldots, N / 2$.

\subsection{Non-Smooth Potentials}

Numerical experiments, reported in [13], demonstrate the effectiveness of KSS methods, in terms of estimates of global error, for problems in which the potential $V(x)$ in (4) and the initial data $f(x)$ are both smooth. When this is not the case, however, accuracy is significantly degraded, especially for larger time steps.

This can be resolved by using a symmetric perturbation, such as the polar decomposition

$$
\begin{aligned}
\mathbf{u}^{T} f(A) \mathbf{v}= & \frac{1}{4 \delta}\left[(\mathbf{u}+\delta \mathbf{v})^{T} f(A)(\mathbf{u}+\delta \mathbf{v})-\right. \\
& \left.(\mathbf{u}-\delta \mathbf{v})^{T} f(A)(\mathbf{u}-\delta \mathbf{v})\right],
\end{aligned}
$$

where $\mathbf{u}$ and $\mathbf{v}$ are real; a generalization to complex $\mathbf{u}$ and $\mathbf{v}$ is straightforward. To see why this is effective, and the original KSS method is not, we examine the effect of perturbation of the initial vectors, both symmetric and unsymmetric, on the recursion coefficients.

For convenience, we define $T\left(L_{N}, \mathbf{u}, \mathbf{v}\right)$ to be the matrix of recursion coefficients produced by the unsymmetric Lanczos method applied to the discretized differential operator $L_{N}$, on a uniform $N$-point grid, with initial vectors $\mathbf{u}$ and $\mathbf{v}$. We set $N=64$ and examine the recursion coefficients for the wave number $\omega=24$. Let $\mathbf{f}$ be the discretized initial data, which we construct so as to be smooth (see [14]). Furthermore, let $\delta=10^{-4}$. We observe the following:

- When we use a smooth potential, then $\left[T\left(L_{N}, \hat{\mathbf{e}}_{\omega}, \hat{\mathbf{e}}_{\omega}+\delta \mathbf{f}\right)-T\left(L_{N}, \hat{\mathbf{e}}_{\omega}, \hat{\mathbf{e}}_{\omega}\right)\right] / \delta$, for $\omega=24$, is

$$
\left[\begin{array}{rr}
1.5807 \mathrm{e}-006 & 1.0388 \mathrm{e}-003 \\
1.0388 \mathrm{e}-003 & 3.2976
\end{array}\right] \text {. }
$$

- When the potential is not smooth, we have, for the same matrix,

$$
\left[\begin{array}{rr}
7.3819 \mathrm{e}-004 & 0.5688 \\
0.5688 & 1.7544 \mathrm{e}+003
\end{array}\right] \text {. }
$$

- Using the same non-smooth potential, the matrix $T\left(L_{N}, \hat{\mathbf{e}}_{\omega}+\mathbf{f}, \hat{\mathbf{e}}_{\omega}+\mathbf{f}\right)-T\left(L_{N}, \hat{\mathbf{e}}_{\omega}-\mathbf{f}, \hat{\mathbf{e}}_{\omega}-\mathbf{f}\right)$ is

$$
\left[\begin{array}{ll}
1.6867 \mathrm{e}-005 & 3.4202 \mathrm{e}-005 \\
3.4202 \mathrm{e}-005 & 1.7100 \mathrm{e}-005
\end{array}\right] \text {. }
$$

Figures 1 and 2 illustrate what happens across all Fourier components. In both figures, the top two plots show the real part of the inverse discrete Fourier transform of the Fourier components computed by approximating individual Riemann-Stieltjes integrals: $\hat{\mathbf{e}}_{\omega}^{H} \exp \left[-i L_{N} \Delta t\right] \hat{\mathbf{e}}_{\omega}$ and $\hat{\mathbf{e}}_{\omega}^{H} \exp \left[-i L_{N} \Delta t\right]\left(\hat{\mathbf{e}}_{\omega}+\delta \mathbf{u}^{n}\right)$ in Figure 1 , and $\left(\hat{\mathbf{e}}_{\omega} \pm\right.$ $\left.\mathbf{u}^{n}\right)^{H} \exp \left[-i L_{N} \Delta t\right]\left(\hat{\mathbf{e}}_{\omega}+\mathbf{u}^{n}\right)$ in Figure 2. As expected, these functions are nearly in agreement with one another.

The bottom left plot shows the real part of the difference quotient of these two functions, and the bottom right plot shows the computed solution in the complex plane. We observe in Figure 1 that when using the asymmetric perturbation, the cancellation does not eliminate highfrequency oscillations, yielding a solution that is much less regular than the initial data, and in fact is highly inaccurate. The symmetric perturbation illustrated in Figure 2 , on the other hand, does not introduce these spurious high-frequency oscillations, yielding a much more acccurate solution, as is demonstrated further in Section 4.

To gain some insight into why the symmetric perturbation induces much less change in the recursion coefficients, we note the following: Let $M_{\mathbf{u}_{0}, \mathbf{v}_{0}}(\mathbf{u}, \mathbf{v})$ : 

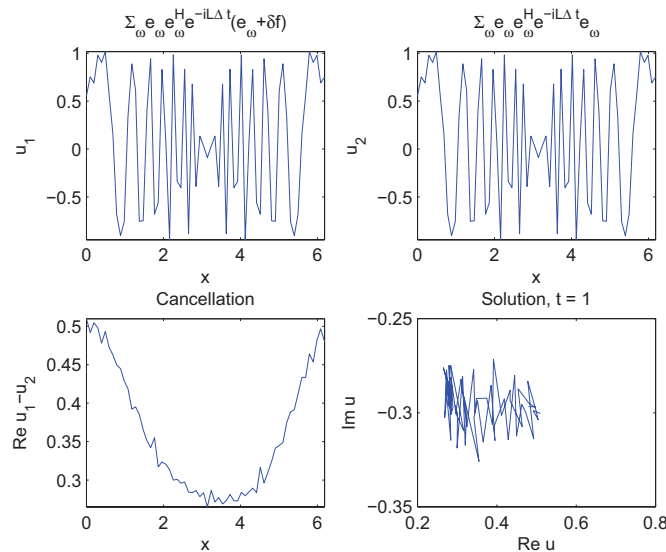

Figure 1: Unsymmetric perturbation
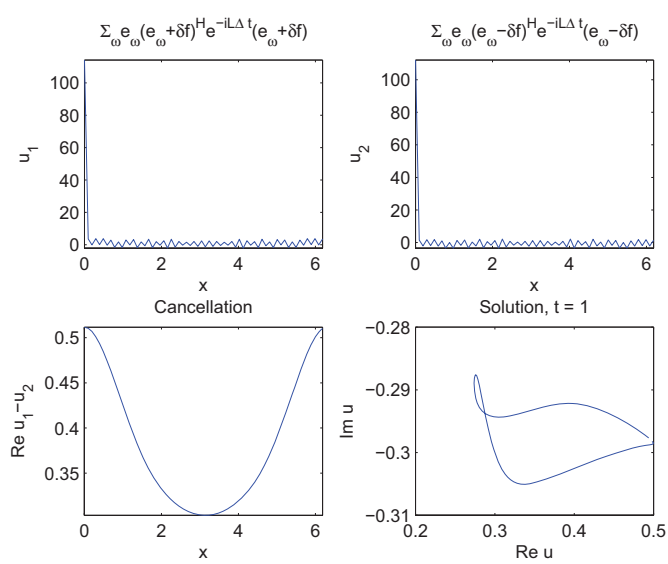

Figure 2: Symmetric perturbation

$\mathbb{C}^{K \times K} \rightarrow \mathbb{C}^{K \times K}$ denote the mapping that transforms $T_{1}$ into $T_{2}$, where $T_{1}$ is the tridiagonal matrix produced by the unsymmetric Lanczos algorithm applied to $L_{N}$ with initial vectors $\mathbf{u}_{0}$ and $\mathbf{v}_{0}$ and $T_{2}$ is the tridiagonal matrix corresponding to initial vectors $\mathbf{u}_{0}+\mathbf{u}$ and $\mathbf{v}_{0}+\mathbf{v}$. Then, we have

$$
M_{\mathbf{u}, \mathbf{u}}(\mathbf{v}, \mathbf{v})=\left[M_{\mathbf{u}+\mathbf{v}, \mathbf{u}+\mathbf{v}}(-\mathbf{v}, \mathbf{0})\right]^{-1} M_{\mathbf{u}, \mathbf{u}}(\mathbf{0}, \mathbf{v}) .
$$

That is, the update of recursion coefficients by a symmetric perturbation of the initial vector is the composition of an update by an unsymmetric perturbation, and the inverse of another update by an unsymmetric perturbation. Therefore, although the two unsymmetric updates, individually, can yield large changes in the recursion coefficients, this view of the process suggests that a certain cancellation takes place, resulting in greater stability in the recursion coefficients from a symmetric perturbation of the initial vector. It should be noted that explicit formulas for the updated coefficients via an unsymmetric perturbation of the right initial vector are given in [15].

\section{Numerical Results}

In this section, we will present numerical results for comparisons between the original KSS method (as described in Section 2) and the symmetrized KSS method described in Section 3. The comparisons will focus on the accuracy of the temporal approximations employed by each method. For convenience, we denote by $\operatorname{KSS}(K)$ the original KSS method with $K$ Gaussian nodes, and by $\operatorname{KSS}-\mathrm{S}(K)$ the symmetrized KSS method with $K$ Gaussian nodes, as in Section 3.2.

We consider a problem in which the potential $V(x)$ has only one continuous derivative. Specifically, its Fourier coefficients $\hat{V}(\omega)$ are chosen, using a procedure described in [14], so that their decay rate is $O\left(|\omega|^{-2}\right)$. It follows that $V^{\prime}(x)$ has high-frequency oscillations. The initial data $f(x)$ is similarly constructed so as to have three continuous derivatives. The case of non-smooth initial data is discussed in [13].

Figure 3 describes the performance of ode23s, the standard 2-node KSS method $\operatorname{KSS}(2)$, and a symmetrized 2-node KSS method KSS-S(2), as described in Section 3.2 , for 64 - and 128-point uniform grids. We observe that compared to the case of a smooth potential, $\operatorname{KSS}(2)$, while maintaining its order of accuracy, exhibits significanly larger error, and substantial degradation of performance when the number of grid points increases. KSS$\mathrm{S}(2)$, on the other hand, performs far better, actually $\mathrm{im}$ proving as the grid is refined, with order of convergence very close to the expected value of 3 .
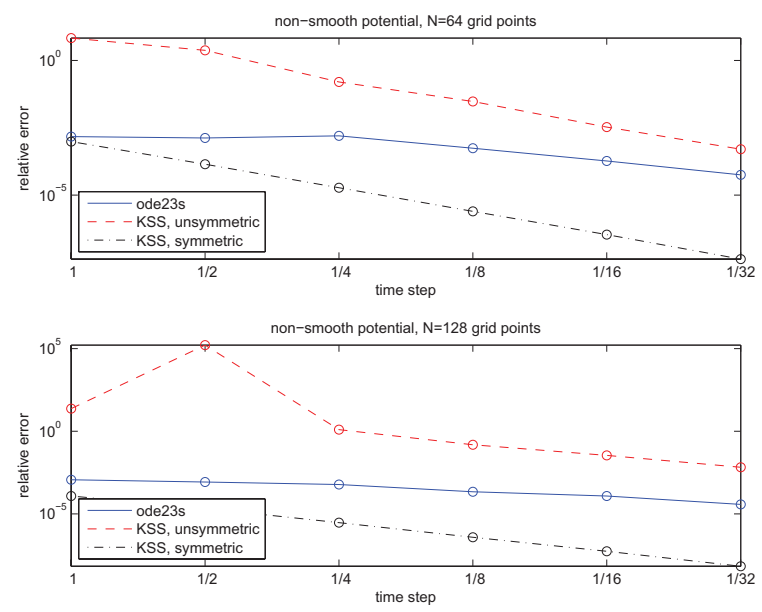

Figure 3: Estimates of relative error in the solution of (1), $(2),(3)$, with $V(x)$ possessing only one continuous derivative, by the MATLAB solver ode23s, the 2-node KSS method KSS(2) with unsymmetric perturbation, and the 2-node KSS method KSS-S(2) with symmetric perturbation, on uniform grids with $N=64$ and $N=128$ points. 
Proceedings of the International MultiConference of Engineers and Computer Scientists $2010 \mathrm{Vol}$ III, IMECS 2010, March 17 - 19, 2010, Hong Kong

\section{Discussion}

While we have used periodic boundary conditions in this paper, it is typical in practical applications of the timedependent Schrödinger equation to use boundary conditions that are more effective at simulating an infinite domain. One such type of boundary condition is a perfectly matched layer (PML), first used by Berenger in [2] for Maxwell's equations. A PML absorbs waves by modifying spatial differentiation operators in the PDE. For example, for absorbing waves that propagate in the $x$ direction, $\frac{\partial}{\partial x}$ is replaced by

$$
\frac{1}{1+\frac{i \sigma(x)}{\omega}} \frac{\partial}{\partial x},
$$

where, as before, $\omega$ represents the wave number, and $\sigma$ is a positive function that causes propagating waves to be attenuated.

In KSS methods, this transformation can be incorporated into the symbol of the operator $L$ during the computation of the recursion coefficients. The dependence of the transformation on both $x$ and $\omega$ makes the efficient application of the transformed operator more difficult, especially in higher space dimensions, but recent work on rapid application of Fourier integral operators (see [3]) can mitigate this concern. Future work will explore the use of PML, taking into account very recent analysis in [17] of the difficulties of PML with inhomogeneous media, and the remediation of these difficulties through adiabatic absorbers.

In summary, we have demonstrated that KSS methods can be applied to the time-dependent Schrödinger equation, and achieve the same order of convergence and stability as for the parabolic problems to which they have previously been applied. Although the Schrödinger equation is more difficult to solve accurately in the case of nonsmooth coefficients, KSS methods can easily be modified to handle this difficulty.

\section{References}

[1] Atkinson, K.: An Introduction to Numerical Analysis, 2nd Ed. (1989) Wiley.

[2] Berenger, J.: "A perfectly matched layer for the absorption of electromagnetic waves", J. Comp. Phys. 114 (1994), pp. 185-200.

[3] Candes, E., Demanet, L., Ying, L.: "Fast Computation of Fourier Integral Operators", SIAM J. Sci. Comput. 29(6) (2007), pp. 2464-2493.

[4] Dahlquist, G., Eisenstat, S. C., Golub, G. H.: "Bounds for the error of linear systems of equations using the theory of moments", J. Math. Anal. Appl. 37 (1972), pp. 151-166.
[5] Golub, G. H.: "Some modified matrix eigenvalue problems", SIAM Review 15 (1973) pp. 318-334.

[6] Golub, G. H.: "Bounds for matrix moments", Rocky Mnt. J. of Math. 4 (1974), pp. 207-211.

[7] Golub, G. H., Meurant, G.: "Matrices, Moments and Quadrature", in Proceedings of the 15th Dundee Conference, June-July 1993, D. F. Griffiths and G. A. Watson (eds.), Longman Scientific \& Technical, 1994.

[8] Golub, G. H., van Loan, C. F.: Matrix Computations, 3rd Ed. (1996) Johns Hopkins University Press.

[9] Golub, G. H., Welsch, J.: "Calculation of Gauss Quadrature Rules", Math. Comp. 23 (1969), 221230 .

[10] Hochbruck, M., Lubich, C.: "On Krylov Subspace Approximations to the Matrix Exponential Operator", SIAM J. Numer. Anal. 34 (1996), pp. 19111925.

[11] Lambers, J. V.: "Derivation of High-Order Spectral Methods for Time-Dependent PDE Using Modified Moments", Electr. Trans. Num. Anal. 28 (2007), pp. 114-135.

[12] Lambers, J. V.: "Implicitly Defined High-Order Operator Splittings for Parabolic and Hyperbolic Variable-Coefficient PDE Using Modified Moments", Intl. J. Comp. Sci. 2(3) (2008), pp. 376-401.

[13] Lambers, J. V.: "Krylov Subspace Spectral Methods for the Time-Dependent Schrödinger Equation with Non-Smooth Potentials", Numer. Alg. 51 (2009), pp. 239-280.

[14] Lambers, J. V.: "Krylov Subspace Spectral Methods for Variable-Coefficient Initial-Boundary Value Problems", Electr. Trans. Num. Anal. 20 (2005), pp. 212-234.

[15] Lambers, J. V.: "Practical Implementation of Krylov Subspace Spectral Methods", J. Sci. Comput. 32 (2007), pp. 449-476.

[16] Moore, W. J.: Schrödinger: Life and Thought, Cambridge University Press (1992).

[17] Oskooi, A. F., Zhang, L., Avniel, Y., Johnson, S. G.: "The failure of perfectly matched layers, and towards their redemption by adiabatic absorbers", Opt. Expr. 16 (2008), pp. 11376-11392.

[18] Shampine, L. F., Reichelt, M. W.: "The MATLAB ODE Suite", SIAM J. Sci. Comput. 18 (1997), pp. 1-22. 